\title{
Gronwall-type integral inequalities with impulses on time scales
}

\author{
Y Peng ${ }^{1,2^{*}}$, Ying Kang ${ }^{2}$, Mingxiu Yuan ${ }^{2}$, Rongfeng Huang ${ }^{2}$ and Liu Yang ${ }^{2}$
}

\author{
* Correspondence: \\ pengyf0803@163.com \\ ${ }^{1}$ School of Mathematics and \\ Statistics, Wuhan University, \\ Wuhan, 430072, People's Republic \\ of China \\ Full list of author information is \\ available at the end of the article
}

\begin{abstract}
In this article, some Gronwall-type integral inequalities with impulses on time scales are investigated. Our results extend some known dynamic inequalities on time scales, unify and extend some continuous inequalities and their corresponding discrete analogues. Some applications of the main results are given in the end of this article. AMS (MOS) Subject Classification 34D09; 34D99, 37M10, 35D05, 49K25, 90C46.
\end{abstract}

Keywords: Time scales, Integral inequalities, Impulse

\section{Introduction}

The theory of time scales, which has recently received a lot of attention, was initiated by Hilger [1] in his Ph.D. thesis in 1988 to contain both difference and differential calculus in a consistent way. Since then many authors have investigated the dynamic equations, the calculus of variations and the optimal control problem on time scales (see [2-11]). At the same time, a few papers have studied the theory of integral inequalities on time scales (see [12-14]).

In this article, we study some Gronwall-type integral inequalities on time scales, which extend some known dynamic inequalities on time scales, unify and extend some continuous inequalities and their corresponding discrete analogues. It is helpful in our result to study dynamic systems and optimal control problem on time scales.

\section{Preliminaries}

For convenience, we present some important theorem on time scales in this section. The approach is based on the ideas in [9] and will be of fundamental importance in following results.

A time scale $\mathbb{T}$ is a nonempty closed subset of $R$. The two most popular examples are $\mathbb{T}=R$ and $\mathbb{T}=Z$. Define the forward and backward jump operators $\sigma, \rho: \mathbb{T} \rightarrow \mathbb{T}$ by

$$
\sigma(t)=\inf \{s \in \mathbb{T} \mid s>t\}, \quad \rho(t)=\sup \{s \in T \mid s<t\}, t \in \mathbb{T},
$$

where, in this definition, we write $\sup \emptyset=\inf \mathbb{T} \equiv a$ and $\inf \emptyset=\sup \mathbb{T} \equiv b$. A point $t \in \mathbb{T}$ is said to be left-dense, left-scattered, right-dense, right-scattered if $\rho(t)=t, \rho(t)$ $<t, \sigma(t)=t, \sigma(t)>t$, respectively. The forward (backward) graininess $\mu: \mathbb{T} \rightarrow[0,+\infty)(v: \mathbb{T} \rightarrow[0,+\infty))$ is defined by $\mu(t)=\sigma(t)-t(v(t)=t-\rho(t))$. Define $P C_{l r d}(\mathbb{T}, R)\left(P C_{r r d}(\mathbb{T}, R)\right)=\left\{x: \mathbb{T} \rightarrow R \mid x, P C_{l r d}(\mathbb{T}, R)\left(P C_{r r d}(\mathbb{T}, R)\right)=\{x: \mathbb{T} \rightarrow R \mid x\right.$ is continuous at right-dense point $t \in \mathbb{T} \backslash \Lambda, x$ exists right limit at $t \in \Lambda$ or left-dense point

(c) 2011 Peng et al; licensee Springer. This is an Open Access article distributed under the terms of the Creative Commons Attribution License (http://creativecommons.org/licenses/by/2.0), which permits unrestricted use, distribution, and reproduction in any medium, provided the original work is properly cited. 
$\|x\|_{P C}=\max \left\{\sup _{t \in \mathbb{T}}\|x(t+)\|, \sup _{t \in \mathbb{T}}\|x(t-)\|\right\}, x$ is left (right) continuous and exists right (left) limit at $t \in \Lambda$ \}. Endowed with norm

$$
\|x\|_{P C}=\max \left\{\sup _{t \in \mathbb{T}}\|x(t+)\|, \sup _{t \in \mathbb{T}}\|x(t-)\|\right\} .
$$

$\left\{P C_{l r d}(\mathbb{T}, X),\|\cdot\|_{P C}\right\}$ and $\left\{P C_{r r d}(\mathbb{T}, X),\|\cdot\|_{P C}\right\}$ are Banach spaces.

Theorem 2.1. (1) Let $f \in L^{1}(\mathbb{T}, R)$. Then

$$
F(t)=\int_{[a, t)} f(\tau) \Delta \tau, t \in \mathbb{T}
$$

implies $F \in C_{r d}(\mathbb{T}, R)$ is $\Delta$-differentiable $\Delta$-a.e. on Tand

$$
F(t)-F(s)=\int_{[s, t)} f(\tau) \Delta \tau \text { for } s, t \in \mathbb{T} .
$$

(2) If $f$ and $g$ are $\Delta$-differentiable $\Delta$-a.e. on $\mathbb{T}$, then

$$
\int_{[s, t)}\left[f^{\Delta}(\tau) g(\tau)+f^{\sigma}(\tau) g^{\Delta}(\tau)\right] \Delta \tau=f(t) g(t)-f(s) g(s) \text { for } s, t \in \mathbb{T} .
$$

The exponential function $e_{p}$ on time scale plays a very important role for discussing dynamic equations on time scales. Define $\Gamma_{1}(\mathbb{T})=\left\{p \in L_{\text {loc }}^{1}(\mathbb{T}, R) \mid 1+\mu(t) p(t) \neq 0\right\}$. For any $p, q \in \Gamma_{1}(\mathbb{T})$, define $p \oplus q=p+q+\mu p q, \ominus p=-\frac{p}{1+\mu p}, p \ominus q=\frac{p-q}{1+\mu q^{q}}$. Further, we can show that $p \oplus q, p \ominus q, \ominus p \in \Gamma_{1}(\mathbb{T})$. Define the generalized exponential function as follows:

$$
e_{p}(t, s)=\exp \left\{\int_{[s, t)} \xi_{\mu(\tau)}(p(\tau)) \Delta \tau\right\} .
$$

Theorem 2.2. Assume that $p, q \in \Gamma_{1}(\mathbb{T})$, then the following hold:

(1) $e_{0}(t, s) \equiv 1, e_{p}(t, t) \equiv 1, e_{p}(t, s) e_{p}(s, r)=e_{p}(t, r), e_{p}(\sigma(t), s)=[1+\mu(t) p(t)] e_{p}(t, s)$;

(2) $e_{p}(t, s)=\frac{1}{e_{p}(s, t)}=e_{\ominus p}(s, t), e_{p}(t, s) e_{q}(\mathrm{t}, s)=e_{p \oplus q}(t, s), \frac{e_{p}(t, s)}{e_{q}(t, s)}=e_{p \ominus q}(t, s)$;

(3) $\left.e_{p}(\cdot, s) \in C_{r d}(\mathbb{T}, R),\left(e_{p}(\cdot, s)\right)^{\Delta}=p(\cdot) e_{p}(\cdot, s), e_{p}(s, \cdot)\right)^{\Delta}=-p(\cdot) e_{p}(s, \sigma(\cdot) \Delta$-a.e. on $\mathbb{T}$.

\section{Main results}

In this section, we deal with Gronwall-type integral inequalities with impulses on time scales. For convenience, we always assume that $f \in L^{1}(\mathbb{T}, R), p_{i}, q_{i} \in L^{1}\left(\mathbb{T}, R^{+}\right)$with $R^{+}$ $=[0,+\infty), 0<\lambda_{i}<1(i=1,2,3,4), \alpha \geq 0, \beta_{k} \geq 0(k=1,2, \cdots, n), x_{t}=\sup _{a \leq s \leq t}|x(s)|$, $x_{\tau_{b}} \sup _{\tau \leq s \leq b}|x(s)|$ in the section.

Theorem A. (1) If $x \in C_{r d}(\mathbb{T}, R)$ satisfies the following inequality

$$
x^{\Delta}(t) \leq p_{1}(t) x(t)+f(t) \Delta-\text { a.e. on } \mathbb{J}
$$


then

$$
x(t) \leq e_{p_{1}}(t, a) x(a)+\int_{[a, t)} e_{p_{1}}(t, \sigma(\tau)) f(\tau) \Delta \tau \text { for all } t \in \mathbb{T} .
$$

(2) If $x \in P C_{l r d}\left(\mathbb{T}, R^{+}\right)$satisfies the following inequality

$$
x(t) \leq \alpha+\int_{[a, t)} p_{1}(\tau) x(\tau) \Delta \tau+\sum_{t_{k}<t} \beta_{k} x\left(t_{k}\right), \forall t \in \mathbb{T},
$$

then

$$
x(t) \leq \alpha \prod_{t_{k}<t}\left(1+\beta_{k}\right) e_{p_{1}}(t, a), \forall t \in \mathbb{T} .
$$

(3) If $x \in P C_{l r d}\left(\mathbb{T}, R^{+}\right)$satisfies the following inequality

$$
x(t) \leq \alpha+\int_{[a, t)} p_{1}(\tau) x(\tau) \Delta \tau+\int_{[a, t)} q_{1}(\tau) x^{\lambda_{1}}(\sigma(\tau)) \Delta \tau+\sum_{t_{k}<t} \beta_{k} x\left(t_{k}\right), \forall t \in \mathbb{T},
$$

then there is a constant $M>0$ such that

$$
x(t) \leq M, \forall t \in \mathbb{T} .
$$

(4) If $x \in P C_{\text {lrd }}\left(\mathbb{T}, R^{+}\right)$satisfies the following inequality

$$
\begin{aligned}
x(t) \leq & \alpha+\int_{[a, t)} p_{1}(\tau) x(\tau) \Delta \tau+\int_{[a, t)} p_{2}(\tau) x_{\tau} \Delta \tau+\int_{[a, t)} q_{1}(\tau) x^{\lambda_{1}}(\sigma(\tau)) \Delta \tau \\
& +\int_{[a, t)} q_{2}(\tau) x_{\sigma(\tau)}^{\lambda_{2}} \Delta \tau+\sum_{t_{k}<t} \beta_{k} x\left(t_{k}\right), \forall t \in \mathbb{T},
\end{aligned}
$$

then there is a constant $M>0$ such that

$$
x(t) \leq M, \forall t \in \mathbb{T} .
$$

(5) If $x \in P C_{l r d}\left(\mathbb{T}, R^{+}\right)$satisfies the following inequality

$$
\begin{gathered}
x(t) \leq \int_{[a, t)} p_{1}(\tau) x(\tau) \Delta \tau+\int_{[a, t)} p_{2}(\tau) x_{\tau} \Delta \tau+\int_{[a, t)} q_{1}(\tau) x^{\lambda_{1}}(\sigma(\tau)) \Delta \tau+\int_{[a, t)} q_{2}(\tau) x_{\sigma(\tau)}^{\lambda_{2}} \Delta \tau \\
+\alpha+\int_{[a, b)} q_{3}(\tau) x^{\lambda_{3}}(\tau) \Delta \tau+\int_{[a, b)} q_{4}(\tau) x_{\sigma(\tau)}^{\lambda_{4}} \Delta \tau+\sum_{t_{k}<t} \beta_{k} x\left(t_{k}\right), \quad \forall t \in \mathbb{T},
\end{gathered}
$$

then there is a constant $M>0$ such that

$$
x(t) \leq M, \forall t \in \mathbb{T} .
$$

Proof. (1) Note that $p_{1} \in L^{1}\left(\mathbb{T}, R^{+}\right)$implies $p_{1} \in \Gamma_{1}(\mathbb{T})$ and $1+\mu(t) p_{1}(t)>0$ for all $t \in \mathbb{T}$. Now

$$
\left[x(t) e_{\ominus p_{1}}(t, a)\right]^{\Delta}=\left[x^{\Delta}(t)-p_{1}(t) x(t)\right] e_{\ominus p_{1}}(\sigma(t), a) \quad \Delta-\text { a.e. on } \mathbb{T} .
$$


Therefore,

$$
x(t) e_{\ominus p_{1}}(t, a)-x(a)=\int_{[a, t)}\left[x^{\Delta}(\tau)-p_{1}(\tau) x(\tau)\right] e_{\ominus p_{1}}(\sigma(\tau), a) \Delta \tau \leq \int_{[a, t)} f(\tau) e_{\ominus p_{1}}(\sigma(\tau), a) \Delta \tau,
$$

that is,

$$
x(t) \leq e_{p_{1}}(t, a) x(a)+\int_{[a, t)} e_{p_{1}}(t, \sigma(\tau)) f(\tau) \Delta \tau \text { for all } t \in \mathbb{T} .
$$

(2) Define

$$
\gamma(t)=\alpha+\int_{[a, t)} p_{1}(\tau) x(\tau) \Delta \tau+\sum_{t_{k}<t} \beta_{k} x\left(t_{k}\right), \forall t \in \mathbb{T} .
$$

By Theorem 2.1, $y$ is $\Delta$-differential $\Delta$-a.e. on $\mathbb{T}$ and

$$
\left\{\begin{array}{l}
y^{\Delta}(t)=p_{1}(t) x(t) \leq p_{1}(t) y(t), \quad t \neq t_{k} \\
\gamma(a)=\alpha, \gamma\left(t_{k}+\right)=\left(1+\beta_{k}\right) y\left(t_{k}\right) .
\end{array}\right.
$$

For $t \in\left[a, t_{1}\right]$, it is obvious to

$$
\gamma(t) \leq \alpha e_{p_{1}}(t, a) .
$$

Further, we have

$$
\gamma(t) \leq \gamma\left(t_{i}+\right) e_{p_{1}}\left(t, t_{i}\right) \leq \alpha\left(1+\beta_{i}\right) \prod_{t_{k}<t_{i}}\left(1+\beta_{k}\right) e_{p_{1}}\left(t_{i}, a\right) e_{p_{1}}\left(t, t_{i}\right)=\alpha \prod_{t_{k}<t}\left(1+\beta_{k}\right) e_{p_{1}}(t, a), \forall t \in\left(t_{i}, t_{i+1}\right] .
$$

Thus,

$$
x(t) \leq \alpha \prod_{t_{k}<t}\left(1+\beta_{k}\right) e_{p}(t, a), \forall t \in \mathbb{T} .
$$

(3) Setting

$$
y(t)=\alpha+\int_{[a, t)} p_{1}(\tau) x(\tau) \Delta \tau+\int_{[a, t)} g_{1}(\tau) x^{\lambda_{1}}(\sigma(\tau)) \Delta \tau+\sum_{t_{k}<t} \beta_{k} x\left(t_{k}\right),
$$

then

$$
\left\{\begin{array}{l}
y^{\Delta}(t)=p_{1}(t) x(t)+g_{1}(t) x^{\lambda_{1}}(\sigma(t)) \leq p_{1}(t) y(t)+g_{1}(t) y^{\lambda_{1}}(\sigma(t)) \Delta-\text { a.e. on } \mathbb{T}, t \neq t_{k} \\
y(a)=\alpha, y\left(t_{k}+\right)=\gamma\left(t_{k}\right)+\beta_{k} x\left(t_{k}\right)=\left(1+\beta_{k}\right) y\left(t_{k}\right) .
\end{array}\right.
$$

Using the conclusion (1), we have

$$
\begin{aligned}
y(t) & \leq \alpha \prod_{t_{k}<t}\left(1+\beta_{k}\right) e_{p_{1}}(t, a)+\prod_{t_{k}<t}\left(2+\beta_{k}\right) \int_{[a, t)} e_{p_{1}}(t, \sigma(\tau)) g_{1}(\tau) \gamma^{\lambda_{1}}(\sigma(\tau)) \Delta \tau \\
& \leq \prod_{t_{k}<t}\left(2+\beta_{k}\right)\left[\alpha e_{p_{1}}(t, a)+\int_{[a, t)} e_{p_{1}}(t, \sigma(\tau)) g_{1}(\tau) \gamma^{\lambda_{1}}(\sigma(\tau)) \Delta \tau\right] .
\end{aligned}
$$


For $t \in \mathbb{T}$, let

$$
h(t)=\alpha e_{p_{1}}(b, a)+e_{p_{1}}(b, a) \int_{[a, t)} g_{1}(\tau) \gamma^{\lambda_{1}}(\sigma(\tau)) \Delta \tau+e_{p_{1}}(b, a) \int_{[a, b)} g_{1}(\tau) \gamma^{\lambda_{1}}(\sigma(\tau)) \Delta \tau,
$$

then $h$ is monotone increasing function and $h(b)=2 h(a)-\alpha e_{p_{1}}(b, a)$,

$$
h^{\Delta}(t)=e_{p_{1}}(b, a) g_{1}(t) \gamma^{\lambda_{1}}(\sigma(t)) \leq \prod_{k=1}^{n}\left(2+\beta_{k}\right)^{\lambda_{1}} e_{p_{1}}(b, a) g_{1}(t) q^{\lambda_{1}}(t), \forall t \in \mathbb{T} .
$$

$\Delta$-integrating from $a$ to $t$, we obtain

$$
h^{1-\lambda_{1}}(t)-h^{1-\lambda_{1}}(a) \leq \rho \int_{[a, t)} g_{1}(\tau) \Delta \tau, \forall t \in \mathbb{T},
$$

where

$$
\rho=\left(1-\lambda_{1}\right) e_{p_{1}}(b, a) \prod_{k=1}^{n}\left(2+\beta_{k}\right)^{\lambda_{1}} .
$$

Now, we observe that

$$
\left(2 h(a)-\alpha e_{p_{1}}(b, a)\right)^{1-\lambda_{1}}-h^{1-\lambda_{1}}(a) \leq \rho \int_{[a, b)} g_{1}(\tau) \Delta \tau .
$$

Letting

$$
\Gamma(z)=\left(2 z-\alpha e_{p_{1}}(b, a)\right)^{1-\lambda_{1}}-z^{1-\lambda_{1}},
$$

then $\Gamma \in C\left(\left[\frac{\alpha e_{p_{1}}(b, a)}{2},+\infty\right), R\right)$ and $\Gamma\left(\frac{\alpha e_{p_{1}}(b, a)}{2}\right)=-\left(\frac{\alpha e_{p_{1}}(b, a)}{2}\right)^{1-\lambda_{1}}<0$,

$$
\lim _{z \rightarrow+\infty} \Gamma(z)=\lim _{z \rightarrow+\infty} \frac{\Gamma(z)}{z^{1-\lambda_{1}}} z^{1-\lambda_{1}}=\lim _{z \rightarrow+\infty}\left[\left(2-\frac{\alpha e_{p_{1}}(b, a)}{z}\right)^{1-\lambda_{1}}-1\right] z^{1-\lambda_{1}}=+\infty .
$$

Using the proof by contraction, one can show that there exists a constant $M>0$ such that $q(a)<M$. Thus,

$$
x(t) \leq y(t) \leq h(a) \leq M, \forall t \in \mathbb{T} .
$$

(4) Setting $\lambda=\max \left\{\lambda_{1}, \lambda_{2}\right\}$,

$$
z(t)=\left\{\begin{array}{l}
1, \quad x(t)<1, \\
x(t), x(t) \geq 1,
\end{array} p(t)=p_{1}(t)+p_{2}(t), g(t)=g_{1}(t)+g_{2}(t), \forall t \in \mathbb{T},\right.
$$

we have

$$
x(t) \leq \alpha+\int_{[a, t)} p(\tau) z_{\tau} \Delta \tau+\int_{[a, t)} g(\tau) z_{\sigma(\tau)}^{\lambda} \Delta \tau+\sum_{t_{k}<t} \beta_{k} z_{t_{k}}, \forall t \in \mathbb{T} .
$$

Furthermore,

$$
z_{t} \leq \alpha+\int_{[a, t)} p(\tau) z_{\tau} \Delta \tau+\int_{[a, t)} g(\tau) z_{\sigma(\tau)}^{\lambda} \Delta \tau+\sum_{t_{k}<t} \beta_{k} z_{t_{k}}, \forall t \in \mathbb{T},
$$


By the conclusion (3), there is a constant $M>0$ such that

$$
x(t) \leq M, \forall t \in \mathbb{T} .
$$

(5) Setting $\lambda=\max \left\{\lambda_{1}, \lambda_{2}, \lambda_{3}, \lambda_{4}\right\}$,

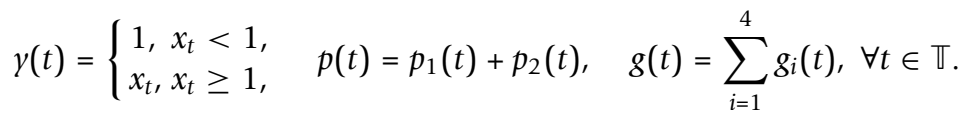

we have

$$
\gamma(t) \leq \alpha+\int_{[a, t)} p(\tau) \gamma(\tau) \Delta \tau+\int_{[a, b)} g(\tau) \gamma^{\lambda}(\tau) \Delta \tau+\sum_{t_{k}<t} \beta_{k} \gamma\left(t_{k}\right) .
$$

By the conclusions (3) and (4), we can show that the conclusion (5) is true. The proof is completed.

In Theorem A, we give some Gronwall-type generalized integral inequalities on time scales. Next, we give some backward Gronwall-type generalized integral inequalities on time scales which can not be directly obtained from Gronwall inequalities.

Theorem B. (1) If $x \in C_{r d}\left(\mathbb{T}, R^{+}\right)$satisfies the following inequality

$$
x(t) \leq f(t)+\int_{[t, b)} p_{1}(\tau) x^{\sigma}(\tau) \Delta \tau,
$$

then

$$
x(t) \leq f(t)+\int_{[t, b)} e_{p_{1}}(\tau, t) p_{1}(\tau) f^{\sigma}(\tau) \Delta \tau \text { for } t \in \mathbb{T} .
$$

(2) If $x \in P C_{r r d}\left(\mathbb{T}, R^{+}\right)$satisfies the following inequality

$$
x(t) \leq \alpha+\int_{[t, b)} p_{1}(\tau) x^{\sigma}(\tau) \Delta \tau+\sum_{t_{k}>t} \beta_{k} x\left(t_{k}\right)
$$

then

$$
x(t) \leq \alpha \prod_{t_{k}>t}\left(1+\beta_{k}\right) e_{p_{1}}(b, t), \forall t \in \mathbb{T} .
$$

(3) If $x \in P C_{r r d}\left(\mathbb{T}, R^{+}\right)$satisfies the following inequality

$$
x(t) \leq \alpha+\int_{[t, b)} p_{1}(\tau) x^{\lambda_{1}}(\tau) \Delta \tau+\int_{[t, b)} g_{1}(\tau) x^{\sigma}(\tau) \Delta \tau,
$$

then there is a constant $M>0$ such that

$$
x(t) \leq M, \forall t \in \mathbb{T} .
$$


(4) If $x \in P C_{r r d}\left(\mathbb{T}, R^{+}\right)$satisfies the following inequality

$$
\begin{aligned}
x(t) \leq & \alpha+\int_{[t, b)} p_{1}(\tau) x^{\lambda_{1}}(\tau) \Delta \tau+\int_{[t, b)} p_{2}(\tau) x_{\tau_{b}}^{\lambda_{2}} \Delta \tau+\int_{[t, b)} g_{1}(\tau) x(\sigma(\tau)) \Delta \tau \\
& +\int_{[t, b)} g_{2}(\tau) x_{\sigma(\tau)_{b}} \Delta \tau+\sum_{t_{k}>t} \beta_{k} x\left(t_{k}\right), \forall t \in \mathbb{T},
\end{aligned}
$$

then there is a constant $M>0$ such that

$$
x(t) \leq M, \forall t \in \mathbb{T} .
$$

(5) If $x \in P C_{r r d}\left(\mathbb{T}, R^{+}\right)$satisfies the following inequality

$$
\begin{gathered}
x(t) \leq \int_{[t, b)} p_{1}(\tau) x^{\lambda_{1}}(\tau) \Delta \tau+\int_{[t, b)} p_{2}(\tau) x_{\tau_{b}}^{\lambda_{2}} \Delta \tau+\int_{[t, b)} g_{1}(\tau) x^{\sigma}(\tau) \Delta \tau+\int_{[t, b)} g_{2}(\tau) x_{\sigma(\tau)} \Delta \tau \\
+\alpha+\int_{[a, b)} g_{3}(\tau) x^{\lambda_{3}}(\tau) \Delta \tau+\int_{[a, b)} g_{4}(\tau) x_{\sigma(\tau)_{b}}^{\lambda_{4}} \Delta \tau+\sum_{t_{k}>t} \beta_{k} x\left(t_{k}\right),
\end{gathered}
$$

then there is a constant $M>0$ such that

$$
x(t) \leq M, \forall t \in \mathbb{T} .
$$

Proof. (1) Define

$$
y(t)=\int_{[t, b)} p_{1}(\tau) x^{\sigma}(\tau) \Delta \tau \text { for } t \in \mathbb{T} .
$$

Then $y(b)=0$ and

$$
\gamma^{\Delta}(t)=-p_{1}(t) x^{\sigma}(t) \geq-p_{1}(t) y^{\sigma}(t)-p_{1}(t) f^{\sigma}(t) \Delta \text { - a.e. on } \mathbb{T} .
$$

Note that,

$$
\left[\gamma(t) e_{p_{1}}(t, b)\right]^{\Delta}=\left[y^{\Delta}(t)+p_{1}(t) \gamma^{\sigma}(t)\right] e_{p_{1}}(t, b) \Delta-\text { a.e. on } \mathbb{T},
$$

therefore,

$$
-\gamma(t) e_{p_{1}}(t, b) \geq-\int_{[t, b)} p_{1}(\tau) f^{\sigma}(\tau) e_{p_{1}}(\tau, b) \Delta \tau .
$$

Moreover, we obtain

$$
x(t) \leq f(t)+\int_{[t, b)} e_{p_{1}}(\tau, t) p_{1}(\tau) f^{\sigma}(\tau) \Delta \tau \text { for } t \in \mathbb{T} .
$$

(2) Setting

$$
\gamma(t)=\alpha+\int_{[t, b)} p_{1}(\tau) x^{\sigma}(\tau) \Delta \tau+\sum_{t_{k}>t} \beta_{k} x\left(t_{k}\right), \forall t \in \mathbb{T} .
$$


then $y$ is $\Delta$-differential $\Delta$-a.e. on $\mathbb{T}$ and

$$
\left\{\begin{array}{l}
y^{\Delta}(t)=-p_{1}(t) x^{\sigma}(t) \geq-p_{1}(t) y^{\sigma}(t), \quad t \neq t_{k} \\
y\left(t_{k}-\right)=y\left(t_{k}\right)+\beta_{k} x\left(t_{k}\right) \leq\left(1+\beta_{k}\right) y\left(t_{k}\right), \\
y(b)=\alpha
\end{array}\right.
$$

For $t \in\left[t_{n}, b\right]$, by the conclusion (1) we have

$$
\gamma(t) \leq \alpha e_{p_{1}}(b, t) .
$$

When $t \in\left(t_{n}, t_{n+1}\right]$, By Theorem 2.2 and the conclusion (1) we also obtain

$$
\gamma(t) \leq \gamma\left(t_{n}-\right) e_{p_{1}}\left(t_{n}, t\right) \leq \alpha\left(1+\beta_{n}\right) e_{p_{1}}(b, t) .
$$

Thus,

$$
x(t) \leq \alpha \prod_{t_{k}>t}\left(1+\beta_{k}\right) e_{p_{1}}(b, t), \forall t \in \mathbb{T} .
$$

(3) Setting $\gamma=(\alpha+1)(\beta+1), \beta=\int_{[a, b)} e_{g}(\tau, a) g(\tau) \Delta \tau$, then

$$
\begin{aligned}
x(t) & \leq \alpha+\int_{[t, b)} p_{1}(\tau) x^{\lambda_{1}}(\tau) \Delta \tau+\alpha \int_{[t, b)} e_{g_{1}}(\tau, t) g_{1}(\tau) \Delta \tau+\int_{[t, b)} e_{g_{1}}(\tau, t) g_{1}(\tau) \int_{[\tau, b)} p_{1}(v) x^{\lambda_{1}}(v) \Delta v \Delta \tau \\
& \leq \gamma+\gamma \int_{[a, b)} p_{1}(\tau) x^{\lambda_{1}}(\tau) \Delta \tau .
\end{aligned}
$$

Letting

$$
h(t)=\gamma+\gamma \int_{[t, b)} p_{1}(\tau) x^{\lambda}(\tau) \Delta \tau+\gamma \int_{[a, b)} p_{1}(\tau) x^{\lambda_{1}}(\tau) \Delta \tau,
$$

then $h$ is monotone descending function and

$$
h^{\Delta}(t) \geq-\gamma p_{1}(t) h^{\lambda}(t) .
$$

$\Delta$-integrating from $t$ to $b$, we obtain

$$
h^{1-\lambda_{1}}(t)-h^{1-\lambda_{1}}(b) \leq\left(1-\lambda_{1}\right) \gamma \int_{[t, b)} p_{1}(\tau) \Delta \tau .
$$

Therefore,

$$
(2 h(b)-\gamma)^{1-\lambda_{1}}-h^{1-\lambda_{1}}(b) \leq\left(1-\lambda_{1}\right) \gamma \int_{[a, b)} p_{1}(\tau) \Delta \tau .
$$

Using the method of the conclusion (3) in Theorem A, one can show that there is a constant $M>0$ such that

$$
x(t) \leq M, \forall t \in \mathbb{T} .
$$

(4) For $t \in \mathbb{T}$, define

$$
z(t)=\left\{\begin{array}{l}
1, \quad x(t)<1, \\
x(t), x(t) \geq 1,
\end{array}\right.
$$


we have

$$
x(t) \leq \alpha+\int_{[t, b)} p(\tau) z_{\tau_{b}} \Delta \tau+\int_{[t, b)} g(\tau) z_{\sigma(\tau)_{b}}^{\lambda} \Delta \tau+\sum_{t_{k}>t} \beta_{k} z_{t_{k b}}, \forall t \in \mathbb{T},
$$

where $\lambda=\max \left\{\lambda_{1}, \lambda_{2}\right\}, p(t)=p_{1}(t)+p_{2}(t) g(t)=g_{1}(t)+g_{2}(t)$. Hence

$$
z_{t_{b}} \leq \alpha+\int_{[t, b)} p(\tau) z_{\tau_{b}} \Delta \tau+\int_{[t, b)} g(\tau) z_{\sigma(\tau)_{b}}^{\lambda} \Delta \tau+\sum_{t_{k}>t} \beta_{k} z_{t_{k b}}, \forall t \in \mathbb{T},
$$

By the conclusion (3), there exists a constant $M>0$ such that

$$
x(t) \leq M, \forall t \in \mathbb{T} .
$$

(5) Setting $\lambda=\max \left\{\lambda_{1}, \lambda_{2}, \lambda_{3}, \lambda_{4}\right\}$,

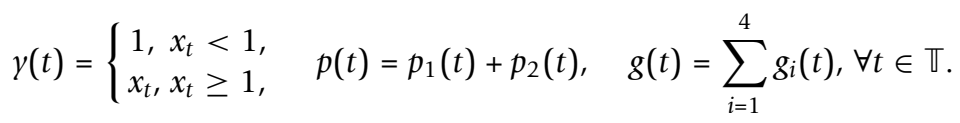

then we have

$$
y(t) \leq \alpha+\int_{[t, b)} p(\tau) y(\tau) \Delta \tau+\int_{[a, b)} g(\tau) \gamma^{\lambda}(\tau) \Delta \tau+\sum_{t_{k}>t} \beta_{k} \gamma\left(t_{k}\right) .
$$

Further, we also can prove that the conclusion (5) is hold. This completes the proof. Remark 3.1: (1) If $\mathbb{T}=R$, then the inequality established in Theorem A reduces to the inequality established by Peng and Wei in [15].

(2) Using our main results, we can obtain many dynamic inequalities for some peculiar time scales. Due to limited space, their statements are omitted here.

\section{Application}

In this section, we present some applications of Theorems A and B to investigate certain properties of solutions of the following impulsive dynamic integral equation

$$
\begin{cases}x^{\Delta}(t)+p(t) x^{\sigma}(t)=f\left(t, x(t), x^{\sigma}(t),(S x)(t),(W x)(t)\right), & t \notin \Lambda, \\ (S x)(t)=\int_{[a, t)} k(t, \tau) s\left(\tau, x(\tau), x^{\sigma}(\tau)\right) \Delta \tau, & t \in \mathbb{T}, \\ (W x)(t)=\int_{[a, b)} m(t, \tau) w\left(\tau, x(\tau), x^{\sigma}(\tau)\right) \Delta \tau, & t \in \mathbb{T} \\ x\left(t_{k}+\right)=x\left(t_{k}\right)+J_{k}\left(x\left(t_{k}\right)\right), & t_{k} \in \Lambda, \\ x(a)=x_{0}, & \end{cases}
$$

where $p \in \Gamma_{1}(\mathbb{T})$.

Definition 4.1: A function $x \in P C_{l r d}(\mathbb{T}, R)$ is said to be a weak solution of (4.1), if $x$ satisfies the impulsive integral equation

$$
x(t)=e_{\ominus p}(t, a) x_{0}+\int_{[a, t)} e_{\ominus p}(t, \tau) f\left(\tau, x(\tau), x^{\sigma}(\tau),(S x)(\tau),(W x)(\tau)\right) \Delta \tau+\sum_{t_{k}<t} e_{\ominus p}\left(t, t_{k}\right) J_{k}\left(x\left(t_{k}\right)\right), t \in \mathbb{T} .
$$

Suppose that:

[F] (1) $k, \quad m \in C_{r d}(\mathbb{T} \times \mathbb{T}, R)$, the functions $f: \mathbb{T} \times R \times R \times R \times R \rightarrow R$, $w: \mathbb{T} \times R \times R \rightarrow R, w: \mathbb{T} \times R \times R \rightarrow R$ are measurable in $t \in \mathbb{T}$ and locally Lipschitz continuous, that is, for any $\rho>0$, there exists a constant $L(\rho)>0$, for all $x_{i}, y_{i}, z_{i}, w_{i} \in$ 
$X$, satisfying $\left\|x_{i}\right\|,\left\|y_{i}\right\|,\left\|z_{i}\right\|,\left\|w_{i}\right\| \leq \rho(i=1,2)$, we have

$$
\begin{aligned}
\left\|f\left(t, x_{1}, y_{1}, z_{1}, w_{1}\right)-f\left(t, x_{2}, y_{2}, z_{2}, w_{2}\right)\right\| & \leq L(\rho)\left(\left\|x_{1}-x_{2}\right\|+\left\|y_{1}-y_{2}\right\|+\left\|z_{1}-z_{2}\right\|+\left\|w_{1}-w_{2}\right\|\right), \\
\left\|s\left(t, x_{1}, y_{1}\right)-s\left(t, x_{2}, y_{2}\right)\right\| & \leq L(\rho)\left(\left\|x_{1}-x_{2}\right\|+\left\|y_{1}-y_{2}\right\|\right), \\
\left\|w\left(t, x_{1}, y_{1}\right)-w\left(t, x_{2}, y_{2}\right)\right\| & \leq L(\rho)\left(\left\|x_{1}-x_{2}\right\|+\left\|y_{1}-y_{2}\right\|\right) .
\end{aligned}
$$

(2) There are constants $0<\lambda_{i}<0(i=1, \ldots, 4)$ and function $q_{1}, q_{2}, q_{3} \in L^{1}\left(\mathbb{T}, R^{+}\right)$ such that

$$
\begin{aligned}
|f(t, x, y, w, z)| & \leq q_{1}(t)\left(1+|x|+|y|^{\lambda_{1}}+|w|+|z|\right), \forall x, y, w, z \in R, t \in \mathbb{T}, \\
|s(t, x, y)| & \leq q_{2}(t)\left(1+|x|+|y|^{\lambda_{2}}\right), \forall x, y \in R, t \in \mathbb{T}, \\
|w(t, x, y)| & \leq q_{3}(t)\left(1+|x|^{\lambda_{3}}+|y|^{\lambda_{4}}\right), \forall x, y \in R, t \in \mathbb{T} .
\end{aligned}
$$

(3) There are constants $\beta_{k} \geq 0$ such that the mapping $J_{k}: R \rightarrow R(k=12 \cdots n)$ satisfies

$$
\left\|J_{k}(x)-J_{k}(y)\right\| \leq \beta_{k}\|x-y\|, \forall x, y \in X .
$$

Theorem C. Under assumption [F], if $p \in \Gamma_{1}(\mathbb{T})$, then the system (4.1) has a weak solution $x \in P C_{l r d}(\mathbb{T}, R)$.

Proof. Define the operator $H$ on $P C_{l r d}(\mathbb{T}, R)$ given by

$$
(H x)(t)=\int_{[a, t)} e_{\ominus p}(t, \tau) f\left(\tau, x(\tau), x^{\sigma}(\tau),(S x)(\tau),(W x)(\tau)\right) \Delta \tau+e_{\ominus p}(t, a) x_{0}+\sum_{t_{k}<t} e_{\ominus p}\left(t, t_{k}\right) J_{k}\left(x\left(t_{k}\right)\right) .
$$

We can first prove that $H: P C_{l r d}(\mathbb{T}, R) \rightarrow P C_{l r d}(\mathbb{T}, R)$ is continuous and compact.

Let $x \in \Upsilon \equiv\left\{x \in P C_{l r d}(\mathbb{T}, R) \mid x=\delta(H x), \delta \in[0,1]\right\}$. When $\delta \neq 0$, set $y=\frac{1}{\delta} x$, if not $y$ $=0$. Note that

$$
\begin{aligned}
|\gamma(t)| \leq & M\left[\left|x_{0}\right|+\left\|q_{1}\right\|_{L^{1}}\left(1+\|k\|\left\|q_{2}\right\|_{L^{1}}+\|m\|\left\|q_{3}\right\|_{L^{1}}\right)+\sum_{k=1}^{n}\left|J_{k}(0)\right|\right] \\
& +M \delta \int_{[a, t)} q_{1}(\tau)|\gamma(\tau)| \Delta \tau+M \delta\|k\|\left\|q_{2}\right\|_{L^{1}} \int_{[a, t)} q_{1}(\tau)\left|\gamma_{\tau}\right| \Delta \tau \\
& +M \delta \int_{[a, t)} q_{1}(\tau)\left|\gamma^{\sigma}(\tau)\right|^{\lambda_{1}} \Delta \tau+M \delta\|k\|\left\|q_{2}\right\|_{L_{1}} \int_{[a, t)} q_{1}(\tau)\left|\gamma_{\sigma(\tau)}\right|^{\lambda_{2}} \Delta \tau, \\
& +M \delta\|m\|\left\|q_{1}\right\|_{L^{1}} \int_{[a, b)} q_{3}(\tau)|\gamma(\tau)|^{\lambda_{3}} \Delta \tau \\
& +M \delta\|m\|\left\|q_{1}\right\|_{L^{1}} \int_{[a, b)} q_{3}(\tau)\left|\gamma^{\sigma}(\tau)\right|^{\lambda_{4}} \Delta \tau+M \sum_{t_{k}<t} \beta_{k}\left|\gamma\left(t_{k}\right)\right| .
\end{aligned}
$$

By Gronwall inequality (5) in Theorem A, there is a constant $M>0$ such that

$$
|\gamma(t)| \leq M, \forall t \in \mathbb{T} .
$$

It follows by Leray-Schauder fixed point theorem, $H$ has a fixed point in $P C_{r d}(\mathbb{T}, R)$, that is, the impulsive integro-differential equation (4.1) has a weak solution $x \in P C_{r d}(\mathbb{T}, R)$. 
For the following backward problem

$$
\left\{\begin{array}{l}
\varphi^{\Delta}(t)+p(t) \varphi^{\sigma}(t)=w\left(t, \varphi(t), \varphi^{\sigma}(t)\right), \quad a \leq t<b, \\
\varphi(b)=\varphi_{1}
\end{array}\right.
$$

we introduce the following assumption:

[W] (1) The function $w: \mathbb{T} \times R \times R \rightarrow R$ is $\Delta$-measurable in $t \in \mathbb{T}$ and locally Lipschitz continuous, i.e. for all $\phi_{1}, \phi_{2}, \psi_{1}, \psi_{2} \in R$, satisfying $\left|\phi_{1}\right|,\left|\phi_{2}\right|,\left|\psi_{1}\right|,\left|\psi_{2}\right| \leq \rho$, we have

$$
\left|w\left(t, \varphi_{1}, \psi_{1}\right)-w\left(t, \varphi_{2}, \psi_{2}\right)\right| \leq L(\rho)\left(\left|\varphi_{1}-\varphi_{2}\right|+\left|\psi_{1}-\psi_{2}\right|\right) \text { for all } t \in \mathbb{T} .
$$

(2) There exist a constant $0<\lambda<1$ and a function $q \in L^{1}(\mathbb{T},[0,+\infty))$ such that

$$
|w(t, \varphi, \psi)| \leq q(t)\left(1+|\varphi|^{\lambda}+|\psi|\right) \text { for all } \varphi, \psi \in R .
$$

We can prove the following result.

Theorem D. Let $p \in \Gamma_{1}(\mathbb{T})$. Under the assumption [W], the backward problem of the nonlinear dynamical equation (4.2) has a unique weak solution $\varphi \in C_{r d}(\mathbb{T}, R)$.

\begin{abstract}
Acknowledgements
The purpose of this paper is to give some Gronwall type integral inequalities with impulses on time scales, which extend some known dynamic inequalities on time scales, unify and extend some continuous inequalities and their corresponding discrete analogues. It is helpful on our result to study dynamic systems and optimal control problem on time scales.

We would like to thank the referees very much for their valuable comments and suggestions. This work is supported by the National Natural Science Foundation of China under grant no. 10961009, the Fok Ying Tung Education Foundation under grant no. 121104 and Introducing Talents Foundation for the Doctor of Guizhou University under grant no. 2010031.
\end{abstract}

\title{
Author details
}

${ }^{1}$ School of Mathematics and Statistics, Wuhan University, Wuhan, 430072, People's Republic of China ${ }^{2}$ Department of Mathematics, Guizhou University, Guiyang, 550025, People's Republic of China

\section{Authors' contributions}

In this paper, YP carried out the main theorem studies, participated in the sequence alignment and drafted the manuscript. YK carried out the Gronwall inequalities studies. MY carried out the backward Gronwall inequalities. LY participated in the Gronwall inequalities studies. RH present some applications on Gronwall inequality in dynamic systems. All authors read and approved the final manuscript.

\section{Competing interests}

The authors declare that they have no competing interests.

Received: 12 December 2010 Accepted: 18 August 2011 Published: 18 August 2011

\section{References}

1. Hilger, S: Analysis on measure chains-a unified approach to continuous and discrete calculus. Results Math. 18, 18-56 (1990)

2. Benchohra, M, Henderson, J, Ntouyas, S: Impulsive Differential Equations and Inclusion. Hindawi Publishing Corporation, New York (2006)

3. Ferreira, RAC, Torres, DFM: Higher-order calculus of variations on time scales. pp. 149-159. Mathematical Control Theory and Finance, Springer, Berlin (2008)

4. Gong, $Y$, Xiang, $X$ : A class of optimal control problems of systems governed by the first order linear dynamic equations on time scales. J Ind Manag Optim. 5, 1-13 (2009)

5. Hilscher, R, Zeidan, V: Weak maximum principle and accessory problem for control problems on time scales. Nonlinear Anal. 70, 3209-3226 (2009). doi:10.1016/j.na.2008.04.025

6. Lakshmikantham, V, Sivasundaram, S, Kaymakcalan, B: Dynamical Systems on Measure Chains. Kluwer Acadamic Publishers, Dordrecht (1996)

7. Liu, H, Xiang, X: A class of the first order impulsive dynamic equations on time scales. Nonlinear Anal. 69, 2803-2811 (2008). doi:10.1016/j.na.2007.08.052

8. Peng, Y, Xiang, X: Necessary conditions of optimality for a class of optimal control problem on time scales. Comp Math Appl. 58, 2035-2045 (2009). doi:10.1016/j.camwa.2009.08.032

9. Peng, Y, Xiang, $X$, Jiang, Y: Nonliear dynamic systems and optimal control problem on time scales, ESAIM Control Optim. Calc Var. Published online by Gambridge University press (2011) 
10. Rynne, BP: $L^{2}$ spaces and boundary value problems on time-scales. J Math Anal Appl. 328, 1217-1236 (2007). doi:10.1016/j.jmaa.2006.06.008

11. Zhan, Z, Wei, W, Xu, H: Hamilton-Jacobi-Bellman equations on time scales. Math Comput Model. 49, 2019-2028 (2009). doi:10.1016/j.mcm.2008.12.008

12. Agarwal, RP, Bohner, M, Peterson, A: Inequalities on time scales: a survey. Math Inequal Appl. 4, 535-557 (2001)

13. Li, WN: Some new dynamic inequalities on time scales. J Math Anal Appl. 326, 363-371 (2007). doi:10.1016/j. jmaa.2006.03.005

14. Li, WN: Some Pachpatte type inequalities on time scales. Comp Math Appl. 57, 275-282 (2009). doi:10.1016/j. camwa.2008.09.040

15. Wei, W, Xiang, X, Peng, Y: Nonlinear impulsive integro-differential equation of mixed type and optimal controls. Optimization. 55(1-2), 141-156 (2006)

doi:10.1186/1687-1847-2011-26

Cite this article as: Peng et al:: Gronwall-type integral inequalities with impulses on time scales. Advances in Difference Equations 2011 2011:26.

\section{Submit your manuscript to a SpringerOpen ${ }^{\circ}$} journal and benefit from:

- Convenient online submission

- Rigorous peer review

- Immediate publication on acceptance

- Open access: articles freely available online

- High visibility within the field

- Retaining the copyright to your article

Submit your next manuscript at $\boldsymbol{\nabla}$ springeropen.com 